\title{
Berichtigung zu der vorstehenden Arbeit: \\ Wilhelm Damköhler, Funktionen geringster Steilheit.
}

Nach Drucklegung meiner Arbeit bemerkte ich folgende Unstimmigkeit:

Der Seite 133 ( $\$ 15$, letzter Absatz) vollzogene Schluß auf die Gültigkeit der Ungleichung

$$
T_{f} \geqq \mathfrak{q}(\mathfrak{c})
$$

für flächenhaft verteilte Punktmengen $\mathfrak{M}$ ist insofern nicht stichhaltig, als es bei der Weite der Kurvenklasse $\mathfrak{C}(\mathfrak{B}, \mathfrak{M})$ sehr wohl geschehen kann, daß auf einer Kurve $c$ die Teilmenge $c^{\prime}$ ein positives Lebesguesches Maß besitzt und daß dennoch in keinem ihrer Punkte von der Funktion $f(x, y) \subset \mathfrak{F}(\mathfrak{B}, \mathfrak{M})$ die Ungleichung

$$
\frac{d f}{d s_{\mathrm{c}}} \geqq \Delta(x, y)
$$

erfüllt wird; denn sobald der Durchschnitt der Menge $c^{\prime}$ mit einer Integralkurve $C$ des Gleichungssystems

$$
\dot{x}=\xi_{1}(x, y), \quad \dot{y}=\xi_{2}(x, y)
$$

auf $C$ nur eine Menge vom Lebesgueschen Maße Null ausschneidet, braucht ja dort (1) nicht zu bestehen. Nun lassen sich aber Beispiele konstruieren, bei welchen eine rektifizierbare Kurve $\mathfrak{c}$ eine "Parallelfaserung" sozusagen monoton durchsetzt, d. h. jede einzelne Faser nur in einem einzigen Punkte schneidet, und bei welcher dennoch die Menge der Punkte $c^{\prime}$, in denen $c$ die Fasern gleichsinnig berührt, auf $\mathfrak{c}$ gemessen ein positives Lebesguesches Maß besitzt.

Um daher die Schlüsse des $\$ 15$ und damit die Aussage des Hauptsatzes in $\S 16$ aufrecht erhalten zu können, müssen wir die Kurvenklasse $\mathfrak{C}(\mathfrak{B}, \mathfrak{M})$ verengen zu der Klasse $\mathfrak{C}^{*}(\mathfrak{B}, \mathfrak{M})$ und haben mithin zu definieren: $\mathfrak{C}^{*}(\mathfrak{B}, \mathfrak{M})$ umfaßt die Gesamtheit aller geschlossenen rektifizierbaren und orientierten Kurven $\mathfrak{c}$ des Bereiches $\mathfrak{B}$, bei welchen der Durchschnitt $c^{\prime}$ derjenigen ihrer Punkte mit der abgeschlossenen Punktmenge $\mathfrak{M}$, in denen die orientierten Linienelemente $d s_{\mathfrak{c}}$ von $\mathfrak{c}$ die Vektoren $\left(\xi_{1}, \xi_{2}\right)$ gleichsinnig berühren, folgenden Aufbau gestattet: $c^{\prime}$ ist die Vereinigung einer Menge $n$ vom auf $\mathfrak{c}$ gemessenen linearen Maße Null und einer Summe höchstens abzählbar unendlich vieler Mengen $c_{i}^{\prime}$, deren jede ganz einer einzelnen Feldkurve $C_{i}$ des Gleichungssystems (2) angehört und auf ihr ein positives oder verschwindendes Lebesguesches Maß haben darf:

$$
\mathfrak{c}^{\prime}=\mathfrak{n}+\sum_{i} \mathfrak{c}_{\mathfrak{i}}^{\prime}
$$


Man überzeugt sich dann leicht, daß für das auf dieser engeren Kurvenklasse $\mathfrak{C}^{*}(\mathfrak{B}, \mathfrak{M})$ definierte Funktional $\mathfrak{q}(\mathfrak{c})$ alle früher bewiesenen Sätze ebenfalls gelten und daß für den Fall, daß $\mathfrak{M}$ auf endlich oder abzählbar unendlich vielen getrennt liegenden Integralkurvenbögen $C$ von (2) verteilt ist, $\mathfrak{C}^{*}(\mathfrak{B}, \mathfrak{M})$ mit der alten Kurvenklasse $\mathfrak{C}(\mathfrak{B}, \mathfrak{M})$ zusammenfällt. Der Hauptsatz des $\S 16$ aber nimmt jetzt folgenden Wortlaut an:

$\mathrm{H}$ auptsatz. Ist die abgeschlossene Punktmenge $\mathfrak{M}$ im Innern des einfach zusammenhängenden Bereiches $\mathfrak{B}$ irgendwie verteilt und liegt auf ihr keine geschlossene Integralkurve des Systems

$$
\dot{x}=\xi_{1}(x, y), \quad \dot{y}=\xi_{2}(x, y),
$$

so enthält die Funktionenklasse $\mathfrak{F}(\mathfrak{B}, \mathfrak{M})$ stets mindestens eine Funktion $\Phi(P)$ kleinstmöglicher Steilheit

$$
T_{\Phi}=T^{\prime}(\mathfrak{B}, \mathfrak{M})
$$

und diese minimale Steilheit läßt sich aus der Betrachtung der Dichtigkeitsverteilungen derjenigen Punkte $\mathfrak{c}^{\prime} \subset \mathfrak{c}$, in denen die Kurven $\mathfrak{c} \subset \mathfrak{C}^{*}(\mathfrak{B}, \mathfrak{M})$ auf $\mathfrak{M}$ die Vektoren $\left(\xi_{1}, \xi_{2}\right)$ gleichsinnig berühren, nach der Formel

$$
T(\mathfrak{B}, \mathfrak{M})=\overline{\mathfrak{q}}(\mathfrak{B}, \mathfrak{M})=\underset{\mathfrak{c} \subset \mathfrak{C}^{*}(\mathfrak{B}, \mathfrak{M})}{\operatorname{obere}} \mathfrak{q}(\mathfrak{c})
$$

berechnen.

N. B. Für. die Betrachtungen des Teiles B (Funkt. Kl. $\mathfrak{F}_{s t}(\mathfrak{B}, \mathfrak{M})$ ) bjeibt es selbstverständlich bei der alten $\operatorname{Kurvenklasse} \mathfrak{C}(\mathfrak{B}, \mathfrak{M})$, die alle geschlossenen rektifizierbaren und orientierbaren Kurven $\mathfrak{c}$ aus $\mathfrak{B}$ umfaßt, Wilche überhaupt $\mathfrak{M}$ schneiden. 